The MCH Navigator: Tools for MCH Workforce Development and Lifelong Learning

Holly Grason

Division of Maternal and Child Health Workforce Development*

Maternal and Child Health Bureau, Health Resources and Services Administration

U.S. Department of Health and Human Services

and

Department of Population, Family and Reproductive Health

Johns Hopkins Bloomberg School of Public Health

615 N Wolfe Street

Baltimore, MD 21205

hgrason@jhsph.edu

Colleen Huebner

Department of Health Services

University of Washington School of Public Health, Seattle, WA

colleenh@u.washington.edu

Alyssa Kim Crawford**

Mathematica Policy Research, Washington, DC

acrawford@mathematica-mpr.com

Marjory Ruderman

Department of Population, Family and Reproductive Health

Johns Hopkins Bloomberg School of Public Health, Baltimore, MD

mruderma@jhsph.edu 
Cathy R Taylor

Gordon E Inman College of Health Sciences and Nursing

Belmont University, Nashville, TN

cathy.taylor@belmont.edu

Laura Kavanagh

Division of MCH Workforce Development

Maternal and Child Health Bureau, Health Resources and Services Administration

U.S. Department of Health and Human Services, Rockville, MD

lkavanagh@hrsa.gov

Anita Farel

Department of Maternal and Child Health

The Gillings School of Global Public Health

University of North Carolina, Chapel Hill

Anita_farel@unc.edu

Joan Wightkin

Department of Community and Behavioral Health

Louisiana State University Health Sciences Center School of Public Health

New Orleans, LA

joanwightkin@yahoo.com 
Deneen Long-White

Department of Health, Human Performance and Leisure Studies Howard University College of Arts and Sciences, Washington, DC dnlong@howard.edu

Shokufeh M Ramirez

Tulane University School of Public Health and Tropical Medicine

New Orleans, LA

sramirez@tulane.edu

Julie Preskitt

Health Care Organization and Policy

School of Public Health, University of Alabama at Birmingham

Meredith Morrissette

Division of MCH Workforce Development

Maternal and Child Health Bureau, Health Resources and Services Administration

U.S. Department of Health and Human Services, Rockville, MD

mmorrissette@hrsa.gov

Arden Handler

University Of Illinois School of Public Health, Chicago, IL handler@uic.edu 
* At the time the manuscript was prepared, Ms. Grason was working at the Health Resources and Services Administration under an IPA arrangement.

** Ms. Crawford was an Intern at the Division of MCH Workforce Development, Maternal and Child Health Bureau while she was working on development of the MCH Navigator. 


\title{
The MCH Navigator: Tools for MCH Workforce Development and Lifelong Learning
}

\begin{abstract}
Purpose. MCH leadership requires an understanding of $\mathrm{MCH}$ populations and systems as well as continuous pursuit of new knowledge and skills. This paper describes the development, structure, and implementation of the MCH Navigator, a web-based portal for ongoing education and training for a diverse $\mathrm{MCH}$ workforce.
\end{abstract}

Description. Early development of the portal focused on organizing high quality, free, web-based learning opportunities that support established learning competencies without duplicating existing resources. An academic-practice workgroup developed a conceptual model based on the MCH Leadership Competencies, the Core Competencies for Public Health Professionals, and a structured review of MCH job responsibilities. The workgroup used a multi-step process to cull the hundreds of relevant, but widely scattered, trainings and select those most valuable for the primary target audiences of state and local MCH professionals and programs.

Assessment. The MCH Navigator now features 248 learning opportunities, with additional tools to support their use. Formative assessment findings indicate that the portal is widely used and valued by its primary audiences, and promotes both an individual's professional development and an organizational culture of continuous learning. Professionals in practice and academic settings are using the MCH Navigator for orientation of new staff and advisors, "just in time" training for specific job functions, creating individualized professional development plans, and supplementing course content. 
Conclusion. To achieve its intended impact and ensure the timeliness and quality of the Navigator's content and functions, the MCH Navigator will need to be sustained through ongoing partnership with state and local MCH professionals and the MCH academic community.

\section{Key Words}

Workforce

Continuing Education

Leadership

Education, Public Health Professional

Title V programs 


\section{The MCH Navigator: Tools for MCH Workforce Development and Lifelong Learning}

Federal and state maternal and child health (MCH or "Title V") agencies are charged with improving the health of all U.S. women, children, and families. To meet this challenge in today's environment of intractable health disparities, difficult economic times, and massive changes in our healthcare delivery system requires resourceful and innovative leaders who can work across disciplines and across systems. Their effectiveness depends also on rapid access to up-to-date empirical evidence and skills training for themselves and for an increasingly changing $\mathrm{MCH}$ workforce.

Historically, MCH workforce training has occurred through programs funded by the Maternal and Child Health Bureau (MCHB) Special Projects of Regional and National Significance (SPRANS) grants to degree-granting graduate training programs and clinical training programs. These grants focused on discipline-specific areas such as nutrition, interdisciplinary clinical programs such as adolescent health, and population health training in Schools of Public Health. In addition, MCHB has funded stand-alone training programs such as the Enhanced Analytic Skills Projects conducted with the Centers for Disease Control and Prevention (CDC), and a variety of MCH certificate programs, workshops, and webinars. While these efforts have produced a strong and capable workforce, they are limited in their reach and their ability to meet the ongoing needs of the entire MCH workforce, including a majority of individuals who enter new job roles with little training in public health (1).

In 2008, the Workforce Development Committee of the Association of Maternal and Child Health Programs (AMCHP) together with the Association of Teachers of Maternal and Child Health (ATMCH) and MCHB assessed state Title V MCH and Children and Youth with Special Health Care Needs (CYSHCN) programs' workforce capacity, training needs, preferred 
modalities, and barriers to training. Survey respondents identified five training needs: translating data into information for needs assessment and planning; designing and conducting program evaluation; systems thinking; writing, especially synthesis and communication of $\mathrm{MCH}$ science for a variety of audiences; and translating policy into organizational plans, structures and programs. The Title $\mathrm{V}$ professionals also indicated a preference for a combination of online and in-person approaches for graduate education ("blended learning") and skill-building workshops for continuing education (CE). Respondents cited a lack of career advancement opportunities, insufficient agency support, and inability to take leave from work as barriers to pursuing graduate education. They also indicated that travel restrictions, inability to obtain release time from work, costs, and lack of geographic access, as the primary barriers to CE (2).

In response to identified workforce needs, a workgroup comprised of $\mathrm{MCH}$ experts developed and launched the MCH Navigator in January 2012. The Navigator is a web-based portal to educational resources relevant to the $\mathrm{MCH}$ and broader public health communities. The workgroup identified, vetted for accuracy and relevancy, and cataloged potential resources by MCH leadership competency, professional skill, and content areas. In sum, the MCH Navigator is a comprehensive, one-stop shop with virtually unlimited potential to provide existing, emerging, and new MCH professionals with access to diverse, free, and high quality online training. This paper describes the development, structure, and implementation of the MCH Navigator to meet the ongoing educational and training needs of a diverse MCH workforce comprised of professionals from multiple disciplinary fields and with a wide range of previous professional employment experiences. The paper concludes with discussion of challenges, trends in use, and other post-implementation findings.

\section{Methods}


Concept development, along with vetting of the content and usability, occurred over a three-year period and involved multiple strategies employed in an iterative process that is typical of developmental, or utilization-focused evaluation $(3,4)$. Below, we describe how the portal was developed, the primary strategies for formative assessment, and the methods employed to collect information on the scope and nature of use and the experiences of those who used the MCH Navigator in this early period.

Design and Development of the MCH Navigator

Development of the MCH Navigator began in 2010 when a small working group composed of graduate public health MCH faculty and state and federal MCH staff elaborated the concept and framework for a learning portal for MCH professionals. From the outset, the workgroup structured the learning system to meet the needs of the heterogeneous $\mathrm{MCH}$ workforce, including staff with different professional responsibilities, professional disciplines, education, work experience, or prior exposure to Title V (Social Security Act) and public health. We aimed to design a dynamic and adaptable system that guides $\mathrm{MCH}$ professionals to resources suited to their learning needs, encourages lifelong learning across all career stages, facilitates self-directed learning, and can be modified to address new and expanding areas of MCH practice. It allows multiple points of entry to address specific learning needs, or a user can develop a more comprehensive learning plan based on the results of a self-assessment of professional skills.

We faced numerous challenges in the design stage, including organizing existing learning resources in a manner congruent with established learning competencies; identifying easily accessible web-based resources available at no cost; and making efficient use of existing resources to avoid duplicating others' efforts. The workgroup developed a conceptual model 
(Figure 1) based on relevant learning competencies and a targeted review of MCH professional job responsibilities. This initial work facilitated the organization of resources into a manageable number of learning areas that are intuitive and easy to navigate. Specifically, we used the 202 items included in the MCH Leadership Competencies (5) and the Core Competencies for Public Health Professionals (6) to identify seven overarching learning areas, each containing multiple subtopics (37 total subtopics). In addition, the workgroup reviewed five types of position descriptions from seven state public health agencies to identify and map seven common areas of work functions to learning areas and subtopics. These learning areas guided the selection and organization of training resources that populate the $\mathrm{MCH}$ Navigator portal. Figure 2 illustrates the mapping of the MCH Leadership competencies to the MCH Navigator's learning areas; we used the same process for mapping the Core Competencies for Public Health Professionals.

Equally important to our goal of selecting resources germane to nationally endorsed $\mathrm{MCH}$ Leadership Competencies and Core Competencies for Public Health Professionals was our goal to assure quality and applicability of training resources selected for the learning portal. We created a rigorous and systematic vetting process that included specific criteria and involved the target user audiences. To accomplish this, workgroup members explored published tools used by other learning management systems to outline criteria for the MCH Navigator that would be both simple and efficient (not burdensome on reviewers), and identified the best available content with respect to currency, accuracy, and relevance of the information, pedagogical approach, and technical aspects of the presentation. The workgroup gave preference to learning opportunities with: an engaging speaker; interactive components; inclusion of additional resources, workbooks or exercises; CE credits offered; and availability in more than one language. We incorporated all of these elements into the selection criteria to assure quality and applicability of posted material. 
The workgroup used these two competency sets and the initial quality criteria to develop a multi-step process to cull the many hundreds of existing, open access, and potentially $\mathrm{MCH}$ relevant training opportunities scattered widely on public websites. We used this process to identify learning opportunities appropriate and acceptable to the primary target audience(s) for posting. The first step was a preliminary examination by MCHB staff to determine whether the learning opportunity was likely to be relevant before trained public health graduate student screeners conducted a more detailed review. Public health faculty screened learning opportunities that passed these two steps, and an expert panel vetted each opportunity to verify its accuracy, quality, and appropriateness for inclusion. Figure 3 illustrates this process.

Formative Assessment Related to Design and Content

Formative assessment involved multiple data collection methods to ensure the learning portal project was functioning as intended. Title $\mathrm{V}$ program staff and Title $\mathrm{V}$ collaborating agencies assessed all MCH Navigator content between 2011 and 2013.

In July 2011, we created a password protected website to host the approximately 120 initial learning opportunities for assessment by key target audiences. A panel of 28 individuals recruited from state, local, and federal $\mathrm{MCH}$ program staff reviewed these postings over a sixweek period. Reviewers made a number of suggestions for improving the website that were addressed in subsequent refinements to the $\mathrm{MCH}$ Navigator, including simplifying the text, streamlining the organization of content, adding a search tool and "getting started" tutorial, providing information about registration requirements on external links, and adding more learning opportunities with interactive elements such as exercises and quizzes. 
Also in July 2011, a seven-person focus group-including five local health agency staff, one state Title V director, and one AMCHP staff member-attending an MCH leadership retreat provided their perspectives on overall utility of the portal. This group recommended that learning opportunities be grouped for specific audiences, particularly “MCH 101" content.

Between June and October 2012, three panels of professionals from local health departments (8 participants), community organizations (4 participants), and state MCH/CYSHCN program (6 participants) reviewed four MCH 101 Orientation Bundles, for: 1) MCH program support staff; 2) mid- to senior-level staff of MCH programs; 3) local health department staff and related programs (e.g., Community Health Centers, home visiting, Healthy Start); and 4) members of advisory committees, community groups, and advocacy organizations. These panelists considered importance to their work, if topics should be added or omitted, and areas where more depth was needed. Across the three panels, over three-fourths of all vetters agreed that the topics they reviewed were important to include in the Orientation Bundles.

Assessment of Early Use

Data on acceptance and use by our $\mathrm{MCH}$ audiences were collected using three primary strategies; these findings are presented in the Results section.

\section{Website Data}

We used Google Analytics ${ }^{\mathrm{TM}}$, a web statistics service, to examine user characteristics and activity for the twelve-month period June 2012-2013.

\section{Webinar Participants}

Potential users completed evaluation questionnaires following webinars presented to various audience groups (e.g., state and local public health practitioners, $\mathrm{MCH}$ faculty, and grantmakers) to introduce them to the MCH Navigator. The same questions were included in each of the 
webinars. Participants used a Likert scale was used to indicate their extent of agreement with the following two statements: " I am confident that I will use the MCH Navigator in my work," and "I am inclined to suggest the MCH Navigator as a resource for others." Webinar participants also provided additional feedback by answering open-ended questions.

Qualitative Data on Use

Answers to open-ended questions of webinar participants, information provided by participants in a conference workshop about professional development using the MCH Navigator, comments from website visitors, and interviews with 11 key informant Title V directors and staff in three states provided additional feedback.

\section{Results}

Learning Portal Content

Roll out of the MCH Navigator began in January 2012. As of August 2013, there are 248 total learning opportunities available through the $\mathrm{MCH}$ Navigator portal. The $\mathrm{MCH}$ Navigator groups the resources by learning area and topic sub-category. Most learning opportunities fit within the topic area of MCH 101 (75 resources), followed by MCH Planning (43 resources) and Leadership (32 resources). The learning opportunities range from short introductory materials of seven minutes to more in-depth modules of over nine hours in duration. Continuing education units in at least one health-related professional discipline are offered for approximately $25 \%$ of the opportunities. Table 1 presents learning opportunities by area and topic sub-category.

The MCH Navigator offers a number of additional resources to assist users of the website: a video tutorial; orientation bundles, with sets of learning opportunities customized for different types of jobs and organizations; self-assessment guide and tools to direct users with specific learning 
needs to resources; and a "Spotlight" section highlighting specific topics such as quality improvement, and the Affordable Care Act.

\section{Overall Use}

During the 12 month period from June 2012 through 2013, approximately 6,800 unique visitors accessed the MCH Navigator website 11,526 times. Of those, 57\% of visitors were new to the MCH Navigator site and 43\% were returning visitors. Of note, visitors used mobile devices, including tablets and smartphones, for 4\% (476) of the total visits. The MCH Navigator made formatting for mobile devices available in December 2012.

Table 2 displays the most popular pages of the MCH Navigator, as determined by the number of page views.

\section{Access}

During the 12-month period, $44 \%$ of users qualified as Direct Traffic, or visitors who typed the web address directly in to their browser. Thirty-five percent of users found the site via a search engine, and $21 \%$ of users arrived at the site by clicking on a link from another website.

The top three landing pages for visitors who typed an $\mathrm{MCH}$ Navigator page directly in to their browser were (1) Home Page, (2) Quality Improvement Spotlight, and (3) MCH 101. When using a search engine to find the MCH Navigator, the top three landing pages were (1) Home Page, (2) Life Course and Social Determinants Framework, and (3) Developing Self and Others.

The top five referral websites (sites where a user clicked on a hyperlink to the MCH Navigator) were (1) mchb.hrsa.gov, (2) jhsph.edu, (3) amchp.org, (4) facebook.com, and (5) mch.spph.ubc.ca.

Geographic Location 
Eighty-seven percent of visits to the MCH Navigator were from sites within the U.S., and there were visits from every U.S. state. Examination of visits made from within Health Resources and Services Administration (HRSA) regions revealed that Region IV states had the highest percentage of visits to the MCH Navigator website (23\%), followed by Regions III (21\%), V (13\%), and IX (10\%). Table 3 presents a breakdown of the number of visits by HRSA regions.

Visits to the MCH Navigator from countries other than the United States comprised 13\% of total visits during the 12-month study period. International usage was most frequent in India (186 visits), followed by Canada (145), the United Kingdom (143), and Australia (47).

Qualitative Feedback from Webinar and Conference Participants and Key Informants Feedback was overwhelmingly positive from two primary target audiences (local and state MCH program staff) participating in webinars about the MCH Navigator. All 66 participants from the webinars responded to questions regarding their likely use. Ninety-one percent agreed with the statement that they will use the MCH Navigator in their work, and 95\% reported being inclined to suggest the $\mathrm{MCH}$ Navigator as a resource for others.

Key informant interviews with 11 Title V directors and managers in Oklahoma, Tennessee, and Maryland (Personal Communications) reflected the views shared by many others that:

- Often, new staff are unfamiliar with MCH or public health in general, creating a need for standardized core content.

- Workforce development is a high priority, but it must meet the training needs of staff with varying roles and functions across multiple programs and agencies at the state and local levels. 
- Agencies need training opportunities that are easy to access in an environment of limited time and funding.

\section{Use of MCH Navigator in Public Health Agencies}

Common mechanisms for use of the $\mathrm{MCH}$ Navigator by public health practice professionals as reported by webinar participants and key informants include both individually driven and agency-wide efforts:

Orientation of new staff and advisors. Webinar participants and key informants cited "MCH 101 " and other foundational content areas in the MCH Navigator as valuable tools for providing orientation and core background knowledge to new hires, interns, advocates, and members of advisory boards.

"Just in time" training for specific job functions. Individuals often reported using the $\mathrm{MCH}$ Navigator to build skills in targeted areas needed for current activities, such as evaluation, needs assessment, and communicating with legislators. Similarly, program directors and managers reported using the $\mathrm{MCH}$ Navigator to provide training for staff involved in specific projects or for those tasked with new functions after agency reorganization or other changes in roles.

\section{Linking professional development to individual needs and performance review. Multiple} respondents reported individually using the MCH Navigator's self-assessment tools to identify their strengths and training needs. They have used the results to communicate their strengths to their supervisors, to help position themselves for promotion or changes in role, and to self-direct professional growth. Many respondents reported that their organizations have incorporated MCH Navigator-based self-assessment and/or learning plans into their performance review systems. Some have developed structured tools and processes to guide and track staff use. One state (TN) developed a Title V Performance Measure specific to use of the MCH Navigator to 
support workforce development.

Supporting a culture of professional development. Respondents provided examples of many additional ways their organizations use the MCH Navigator to promote an environment of professional development. Multiple respondents said they give presentations on the $\mathrm{MCH}$ Navigator to regional or local MCH and CYSHCN program managers. Another common strategy is to build use of the MCH Navigator into regular team, management, or division-level meetings, or to establish regular "lunch and learn" sessions.

Use of MCH Navigator in Academic Settings

Individuals from institutions of higher education affiliated with Schools of Public Health, MCHBfunded Training Programs, and community colleges who responded to webinar feedback surveys and the website comments page reported using the MCH Navigator in a variety of ways to expand curricula, enhance faculty capacity, and orient potential applicants and trainees to $\mathrm{MCH}$ principles.

Introducing principles of public health and the field of maternal and child health. $\mathrm{MCH}$

Training Programs have used the "MCH 101" content to educate potential applicants about the field of MCH. Similarly, they report using the portal to orient clinical or other disciplinary trainees without previous exposure to population health. One MCH Program is creating an orientation bundle that draws from the MCH Navigator's MCH 101, Epidemiology, and MCH Planning Cycle sections.

Supplementing course content. Respondents report using the MCH Navigator to integrate online resources into existing courses, to provide additional training to students with specific interests or needs, to prepare students for courses when they lack prerequisites, and to create 
new learning opportunities. One faculty member reported using the MCH Navigator to provide the necessary MCH background knowledge for epidemiology students taking a reproductive and perinatal epidemiology class.

Providing knowledge and skills for internships and field placements. Individual students have reported using the MCH Navigator to build skills they need for practicums and internships. The Nashville (TN) public health agency uses the MCH Navigator to augment the field-based learning of summer interns who come from public health programs without core MCH courses.

Enhancing faculty capacity. Faculty reported using the self-assessment component of the MCH Navigator on their own or as part of a Department-wide assessment for faculty members. One faculty member who provides technical assistance to public health agencies recommended using the MCH Navigator to deliver background knowledge before engaging in site visits.

\section{Discussion}

Evidence to date indicates that the MCH Navigator on-line training portal is meeting a need for real-time training to augment face-to-face engagement in learning, such as conferences, workshops, and formal on-campus graduate programs. Even in its earliest stages of development, state and local public health $\mathrm{MCH}$ professionals and programs value and widely use the portal for their training and continuing education needs.

Many common themes related to the unique value of the MCH Navigator emerged from key informant interviews, surveys of webinar and workshop participants, initial reviewers, and website comments.

- The MCH Navigator brings together core MCH training in one place; users appreciate not having to search for these materials on their own. 
- Structured use of the MCH Navigator, either by individuals or throughout organizations, instills a sense of the importance of professional development.

- Respondents value the ability to identify and access learning opportunities when specific tasks require them, as well as to enhance performance in their ongoing roles.

- Free, online training is critical in a time of severely limited time and funding.

- The MCH Navigator is likely to be useful in the public health agency accreditation process, which requires them to provide public health competency based training and to document staff participation.

The most common negative feedback about the MCH Navigator relates to technical challenges, such as inability to connect to a host site or open an audio file. Additionally, some respondents noted that the need to register with multiple external sites in order to access some of the learning opportunities was inconvenient.

Formative assessment findings point to the MCH Navigator's role in promoting leadership development, supporting an individual sense of agency in professional development, and an organizational culture that values and promotes professional development. Although online resources alone are not sufficient for leadership or professional development, access to such resources provides a framework and catalyst for new knowledge and skills that must be linked actively and thoughtfully to day-to-day work. The MCH Navigator has been developed at a time when it is recognized that MCH staff can no longer rely exclusively on agency resources in order to obtain and retain the $\mathrm{MCH}$ expertise needed in our current highly complex and rapidly changing healthcare environment. In fact, state program directors already are exercising their leadership roles and skills by devising tools and implementing strategies that embed 
performance development and the use of the MCH Navigator in program operations. In addition, as formal MCH professional education and training programs continue in their efforts to instill leadership efficacy among their trainees, the MCH Navigator is well-positioned to serve as the "go-to" site for life-long MCH learning. To do so effectively will require engaging students in $\mathrm{MCH}$ training programs in ways that encourage them to view the MCH Navigator as a source of professional development throughout their careers.

While the Navigator is now robustly populated with quality and relevant content (primarily individual presentations), there is a need from both a pedagogical and an audience preference perspective to provide context and tools for learners so that the learning opportunities are more than just sessions of "listening to" individual informational presentations. Moving beyond this more traditional format may be more effective at imparting more nuanced skills and engaging professionals with different learning styles. Some initial work on packaging content and offering guidance on group sessions has occurred, but future approaches may entail providing opportunities for learners to engage with each other and apply emerging skills and knowledge in real time, employing strategies such as on-line learning groups (peer led or moderated by expert faculty) and/or longitudinal workshops that blend Navigator postings with in-person sessions at annual $\mathrm{MCH}$ conferences.

By design, public health principles historically formed the foundation for $\mathrm{MCH}$ programs, yet the field of public health is undergoing significant changes in the face of healthcare reform. Evolving standards of care and critical demands for cost containment and systems re-design are driving the need to integrate new technologies and strategies into public health programs and practice. If $\mathrm{MCH}$ is to remain a key player in these reforms, similar program modifications will be required. The MCH discipline should identify and systematically promulgate new professional 
MCH competencies, such as those related to quality management, informatics and systems integration. This will likely increase the pressure to expand the MCH Navigator content focus beyond the fundamentals of public health maternal and child health. As noted earlier, a major impetus for development of the portal was to streamline overwhelming volumes of training material. Thus, future MCH Navigator stewards must respond to legitimate demands to integrate relevant, new material while avoiding mission creep and recreating the very problems we designed it to address.

This paper describes the MCH Navigator in its formative proof of concept phase. To achieve its intended impact, the MCH Navigator will need to be sustained through financial, technological, and ongoing partnership strategies. MCHB has taken initial steps in this regard, identifying funding to support an institutional project office, and awarding a cooperative agreement to Georgetown University for the 2013-2016 period. To provide a technical platform for the $\mathrm{MCH}$ Navigator capable of addressing target audience learning management system (LMS) needs, MCHB partnered with the Bureau of Health Professions (BHPr) to establish the HRSA affiliate of TrainingFinder Real-time Affiliated Integrated Network (TRAIN), which is widely used across the public health field and includes key features desired by the MCH community, such as transcripts and competency tracking, learning assessments, course rating tools, robust data for ongoing evaluation and quality improvement, and potential for a single registration system. Integrating with TRAIN makes using the MCH Navigator seamless for state and local MCH staff, increasing the likelihood that professional development generally, and the use of this specialized MCH training portal specifically, will become part and parcel of the work of MCH professionals and not regarded as an added, burdensome responsibility. Stakeholder buy-in and ongoing involvement of the $\mathrm{MCH}$ community, particularly the primary target 
audiences, will be key for sustainability. Without the spirit of shared ownership, the MCH Navigator could run the risk of being another marginal resource. Ongoing state and local MCH practitioner input and evaluation will be critical to ensure that it is relevant, useful, and used. Continued partnership with the MCH public health academic community can sustain the currency and quality of the content. Strategies for operationalizing such involvement might include securing an expert consultant panel comprised of ten to twelve state and local $\mathrm{MCH}$ practitioners and $\mathrm{MCH}$ public health academic faculty who would provide oversight to the management of the project now located at Georgetown University. Collaborations drawing on these stakeholder groups' expertise and resources to develop training content to fill existing and newly emerging knowledge and skill competency gaps also would serve to bolster the scope of stakeholder buy-in critical to sustainability.

\section{Limitations}

The available statistics provide useful information about visits to the MCH Navigator website but have some limitations. Most notably, they do not provide information about use of the trainings themselves. Because the MCH Navigator is a centralized portal for accessing learning opportunities hosted on external websites, Google Analytics cannot reveal which trainings are accessed, how long people spend on them, and/or whether modules are completed. Additionally, a greater numbers of visits to any particular section of the MCH Navigator may imply that the content is more popular or desired, but also could reflect the current number of offerings or level of difficulty with accessing particular modules. Finally, selected groups of MCH professionals, not a structured analytic effort, provided qualitative information about current uses of the $\mathrm{MCH}$ Navigator. 


\section{Conclusion}

MCH leadership involves a set of specific qualities and characteristics, including understanding MCH values, mission and goals, possession of core knowledge of MCH populations and needs, and pursuit of new knowledge and skills throughout one's career (5). Contemporary demands for $\mathrm{MCH}$ leadership are substantial and urgent. The $\mathrm{MCH}$ Navigator is a promising means to address this demand and to support MCH professionals as they lead to meet the challenges before them. 


\section{Acknowledgments}

We are grateful to the numerous MCH colleagues who contributed their time and expertise to the development of the MCH Navigator in multiple ways, including by participating in focus groups, serving on vetting panels, and disseminating information about the new resource. There are too many to identify individually; it has truly been a "MCH community effort." That said, we want to extend special thanks to three colleagues who participated in the MCH Navigator Workgroup along with us - Beverly Mulvihill, Jeanette Magnus, and Violanda Grigorescu. We also wish to acknowledge and thank the students who engaged with us to get the work of transforming ideas into reality done. They are: Susannah Anderson; Ashley Belton; Rachel Brzezinski; Andria Cornell; Sara Daleiden; Margaret Hicken; Joseph Lee; Jessica Nelson; Todd Schrecengost; Heather Skanes; Nicole Steffens; and Kathy Vetter. Finally, we share our appreciation for the skill and patience of the technical team - Randy Miller and Robert Peck - who translated the paper version of the portal into the MCH Navigator website. The views expressed are the authors' and not necessarily those of the Health Resources and Services Administration or the US Department of Health and Human Services. 


\section{References}

(1) Levi, J., Kaiman, S., Juliano, C., \& Segal, L. (2008). Blueprint for a healthier America:

Modernizing the federal public health system to focus on prevention and preparedness.

Washington, DC: Trust for America's Health. http://healthyamericans.org/assets/

files/Blueprint.pdf.

(2) Grason H, Kavanagh L, Dooley S, Partelow J, Sharkey A, Bradley KJ and Handler A. (2012).

Findings from an assessment of state Title V workforce development needs. Maternal Child

Health J, 16 (1), 7-20.

(3) Patton, MQ. (1994). Developmental evaluation. American Journal of Evaluation 15: 311-319.

(4) Patton, MQ (1997). Utilization-focused evaluation: the new century text. $3^{\text {rd }}$ Ed. Thousand Oaks, California: Sage Publications: 1997.

(5) Maternal and Child Health Leadership Competencies. Version 3.0. June 2009. http://leadership.mchtraining.net/?page id=184. Accessed 5 Sept 2013.

(6) The Council on Linkages Between Academia and Public Health Practice. Core Competencies for Public Health Professionals. May 2010. http://www.phf.org/programs/corecompetencies. Accessed 5 Sept 2013. 
Table 1. MCH Navigator Content, Organized by Learning Area, August 2013

Learning Area Topic Sub-Categories

Number of

Learning

Opportunities

MCH 101

Population Health 6

MCH Population, Mission \& Principles 29

Title V History \& Legislation $\quad 6$

Title V Implementation 19

Accountability 4

Additional Compilation/Resources $\quad 11$

Total $\quad 75$

MCH Conceptual

Models

Ecological Model 2

Child \& Adolescent Development $\quad 5$

Weathering Hypothesis 1

Life Course Framework 9

Behavior Change Theories and Models 1

Quality of Life Measurement Models 0

Total

Management

Theories and Principles 3

Human Resources 11

Skills 11

Additional Compilations/Resources $\quad 1$

Total 
Communication

$\begin{array}{ll}\text { Fundamentals: Concepts \& Skills } & 7\end{array}$

Scientific Writing $\quad 2$

Grant Writing 3

Translational Writing 4

Public Speaking/Presentations 3

Working with the Media \& Social Media 9

New Media/ $\quad 0$

Communication Technologies

Data Presentation $\quad 1$

Additional Compilations/Resources 1

Total $\quad 30$

Epidemiology

Basic for "Data Users” 16

$\begin{array}{ll}\text { Advanced for "Data Makers" } & 7\end{array}$

Additional Compilations/Resources 1

Total 24

Leadership

Concepts and Theories 4

Developing Vision \& Creating Clarity 1

Developing \& Managing Teams 5

Conflict Negotiation 4

Organizational Change 3

Developing Self \& Others $\quad 10$

$\begin{array}{ll}\text { Ethics } & 5\end{array}$

Total

$\begin{array}{lll}\text { MCH Planning Cycle Overview } & 4\end{array}$ 
Assessment

Program Planning

Program Implementation

Program Evaluation

Policy Development \& Analysis
6

12

9

7

5

Total

TOTAL

248 
Table 2: Top 10 Most Accessed Pages for the MCH Navigator Website

1. Home page

2. $\mathrm{MCH} 101$

3. MCH 101 Orientation Bundles

4. MCH Conceptual Models

5. Population Health

6. Leadership

7. Key to Leadership Competencies

8. Learning Resources Landing Page

9. Self Assessment

10. Quality Improvement 
Table 3. Number of Visits to the MCH Navigator Website by HRSA Regions, June 2012-2013

\begin{tabular}{|l|l|l|}
\hline HRSA Region & States & Number of Visits \\
\hline I & CT, MA, ME, NH, RI, VT & 500 \\
\hline II & NJ, NY, PR, VI & 367 \\
\hline III & DC, DE, MD, PA, VA, WV & 2,060 \\
\hline IV & AL, FL, GA, KY, MS, NC, SC, TN & 2,272 \\
\hline V & IL, IN, MI, MN, OH, WI & 1,280 \\
\hline VI & LA, NM, OK, TX & 592 \\
\hline VII & IA, KS, MO, NE & 513 \\
\hline VIII & CO, MT, ND, SD, UT, WY & 528 \\
\hline IX & AS, AZ, CA, FM, GU, HI, MH, MP, NV, PW & 946 \\
\hline X & AK, ID, OR, WA & 835 \\
\hline
\end{tabular}

